\title{
Coordinating transmit power and carrier phase for wireless networks with multi-packet reception capability
}

\author{
Wooyeol Choi ${ }^{1}$, Taewoon Kim² ${ }^{2}$ Daeyoung Park ${ }^{3}$, Heung-No Lee ${ }^{1}$ and Hyuk Lim 1,4*
}

\begin{abstract}
Driven by advances in signal processing and multiuser detection (MUD) technologies, it has become possible for a wireless node to simultaneously receive multiple signals from other transmitters. In order to take full advantage of MUD in multi-packet reception (MPR) capable wireless networks, it is highly desirable to make the compound signals from multiple transmitters more separable on its constellation at the receiver by coordinating both the transmit power level and carrier phase offsets of the transmitters. In this article, we propose a feedback-based transmit power and carrier phase adjustment scheme that estimates the symbol energy and the carrier phase offset for each transmitter's received signal, computes the optimal received power level and carrier phase shift to maximize the minimum Euclidean distance between the constellation points, and finally feeds the optimal transmit power level and phase shift information back to the transmitters. We then evaluate the performance of the proposed transmit power and carrier phase adjustment scheme and subsequently show that the proposed scheme significantly reduces the error probability in a multiuser communication system having MPR capability.
\end{abstract}

\section{Introduction}

In conventional wireless networks, each receiver is only capable of decoding signals from one transmitter at a time; referred to as single-user detection (SUD). In SUD, when a mixed signal from multiple transmitters is sensed, the receiver typically discards the signal and treats it as a collision. However, signal processing technology has rapidly evolved, and compound signals from multiple transmitters have become decodable at the receiver side $[1,2]$. To effectively decode multiple signals in a multiple access environment, multiuser detection (MUD) can be used. In [2], the optimum multiuser detector has a computational complexity that increases exponentially with the number of active users. Therefore, several suboptimum detectors have been proposed in order to achieve a performance comparable with that of the optimum detector while

\footnotetext{
*Correspondence: hlim@gist.ac.kr

${ }^{1}$ Gwangju Institute of Science and Technology, Gwangju 500-712, Republic of Korea

${ }^{4}$ Department of Nanobio Materials and Electronics, Gwangju Institute of

Science and Technology, Gwangju 500-712, Republic of Korea

Full list of author information is available at the end of the article
}

maintaining a low complexity. The decorrelating detector [3], the decision feedback detector [4], the minimum mean squared error (MMSE) [5], and multistage detectors [6] are examples of suboptimum multiuser detectors. Some of these multiuser detectors are also suitable for blind adaptive implementations, in which information about the interfering users (such as their powers and signature sequences) is not needed for the construction of the receiver filter of a desired user. A blind adaptive implementation of an MMSE multiuser detector is given in [7], and blind adaptive decorrelating detector implementations are shown in $[8,9]$.

Since MUD technology permits simultaneous packet reception from multiple sources, compound signals, which were previously treated as a collision event in conventional wireless networks, are now preferred for their ability to enhance the achievable throughput performance [10-16]. However, how to take advantage of the MUD technique and how to adjust its tunable parameters in designing the medium access control (MAC) for multipacket reception (MPR) capable wireless networks and maximize the achievable throughput have yet to be sufficiently studied.

\section{Springer}

2013 Choi et al licensee Springer. This is an Open Access article distributed under the terms of the Creative Commons

Attribution License (http://creativecommons.org/licenses/by/2.0), which permits unrestricted use, distribution, and reproduction in any medium, provided the original work is properly cited. 
Considering the error-prone nature of the wireless medium, the symbol separation and decoding of a mixed signal are primarily influenced by channel conditions and characteristics. To this end, several studies have attempted to overcome channel effects by means of carrier phase error correction [17-20]. Steendam et al. [17] investigated the effects of carrier phase offsets on a low-density parity-check (LDPC) coded system, and then proposed a maximum likelihood (ML)-based carrier phase synchronization algorithm that exploits the posterior probabilities of the data symbols. Similarly, Zhang et al. [18] proposed an a priori probability aided carrier phase estimation for turbo decoding. They showed that the physical (PHY) layer technique provides a reliable carrier phase estimation that approaches the Cramer-Rao bounds at a very low signal-to-noise ratio (SNR). Harshan et al. $[19,20]$ then identified the problem of maximizing the capacity region between two users for a Gaussian multiple access channel (GMAC). By performing a rotation on one of the sets in such a way that the error probability is minimized, the capacity gain can be maximized. Compared to Harshan's study, our study is applicable to a more general and complex condition.

Even though we consider a feedback-based adjustment using a centralized control to coordinate both the transmit power and carrier phase of the transmitters, a distributed method for achieving the phase coherence of transmitters was also proposed. In [21], the phase alignment for distributed transmit beamforming was independently performed at each transmitter using minimal feedback from the receiver. Through feedback based on the SNR from the receiver, each transmitter decides whether their applied random phase is kept or not, and this iterative process is repeated until all transmitters converge to phase coherence. In [10], a carrier phase adjustment scheme that attempts to maximize the minimum Euclidean distance among the constellation points was proposed for multiple access networks with multi-packet reception capability. Because this scheme simply assumes that the received power levels from multiple transmitters are the same at the receiver, it adjusts the carrier phase offsets of transmitters, but does not coordinate the transmit power levels of transmitters.

In this article, we propose a MAC/PHY cross-layer approach for enhancing the separation and decoding performance of compound signals on an additive white Gaussian noise (AWGN) channel with phase noise effects. Specifically, this article focuses on more complex and realistic scenarios than our previous study in [10]. In this article, we consider the coordination of not only the transmitters' carrier phase offsets but also their transmit power levels. In this system, a receiver with MPR capability performs multiuser detection and then estimates the symbol energy and the carrier phase offset for each transmitter's signal from the compound signals. Next, the receiver piggybacks the optimal transmit power level and carrier phase shift, which is the difference between the estimated carrier phase offset and an optimal carrier phase offset, to the corresponding transmitters so that they can adjust their transmit power and carrier phase offset to the optimal value when transmitting signals. To determine the optimal transmit power level and carrier phase shift, we formulate an optimization problem in order to maximize the minimum Euclidean distance between the constellation coordinates of the compound signals. We subsequently evaluate the performance of the proposed transmit power level and carrier phase adjustment scheme and compare it to that of the no adjustment case for QPSK and 8PSK with 2-4 transmitters. The simulation results show that the proposed scheme significantly reduces the error probability for all cases investigated in our simulation scenarios.

The remainder of this article is organized as follows. Section 2 describes the system model on which the proposed scheme is based, and the motivation that initiated this study. Section 3 then explains the mathematical basis and detailed procedures of the proposed scheme. The performance evaluation is carried out in Section 4, and we finally conclude this article in Section 5.

\section{System model and motivation}

We consider a simple MAC protocol for an uplink singlecell system that is coordinated by a base station (BS) having MPR capability. Because all transmitters in a cell are associated with and continuously communicate with the corresponding BS in the cell, symbol level synchronization at the BS is assumed to be possible in this study. In this multiple access communication system, all transmitters that want to send a data frame are required to transmit a request-to-send (RTS) frame to their intended receiver, which is then responsible for coordinating the packet transmissions among the competing transmitters. On receiving multiple RTS frames, the receiver broadcasts a clear-to-send (CTS) frame, which includes the set of transmitters that are permitted to transmit. We will use this CTS frame to inform the transmitters of the feedback information (optimal transmit power levels and carrier phase shifts), which are calculated by the proposed transmit power and carrier phase adjustment scheme ${ }^{\mathrm{a}}$.

Figure 1 shows the block diagram for the proposed transmit power and carrier phase adjustment scheme. Each transmitter sends a signal to its receiver, and due to the AWGN, the compound signals $y$ from multiple transmitters at the receiver is given by

$$
y=\sum_{i=1}^{N} g_{i} p_{i}^{t} e^{j \theta_{i}} x_{i}+n
$$




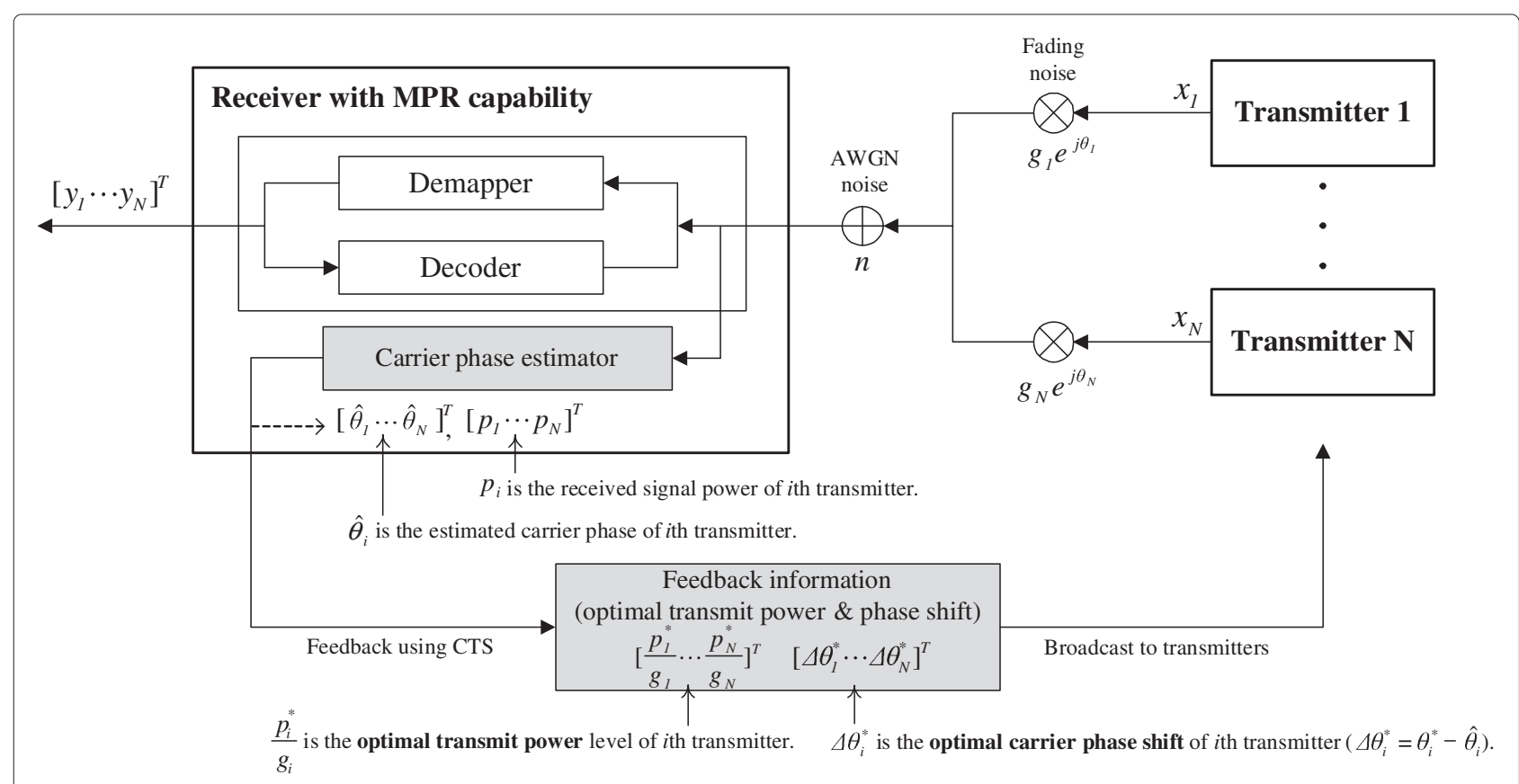

Figure 1 Structure of the proposed scheme. Optimal transmit power level and carrier phase adjustment scheme for a multiple access communication system with multi-packet reception capability.

where $N$ is the number of transmitters, $g_{i}$ is the channel gain of the $i$ th transmitter, $p_{i}^{t}$ is the transmit power level of the $i$ th transmitter, $\theta_{i}$ is the carrier phase of the $i$ th transmitter, $x_{i}$ is the sequence of independent and identically distributed (i.i.d.) equiprobable $i$ th transmitter data symbols, and $n$ is the complex valued AWGN channel noise.

In the figure, the signals transmitted from multiple transmitters pass through the channel with AWGN and fading, in which the signal waveforms are changed by the channel gain $g_{i}$ and AWGN noise $n$. When the compound signals are received, the receiver can estimate the received signal power $p_{i}$ and carrier phase $\hat{\theta}_{i}$. Using the values of received power and estimated carrier phase, the proposed scheme then solve the optimization problem to obtain the optimal received power $p_{i}^{*}$ and carrier phase $\theta_{i}^{*}$. To reduce the feedback burden, the receiver broadcasts the optimal transmit power (optimal received power divided by channel gain, $\frac{p_{i}^{*}}{g_{i}}$ ) and carrier phase shift (difference between optimal carrier phase and estimated carrier phase, $\Delta \theta_{i}^{*}=\theta_{i}^{*}-\hat{\theta}_{i}$ ) to the multiple transmitters. In this way, the multiple transmitters can apply the fed-back value to the next transmission of each transmitter, and then the receiver can achieve a higher performance in the communication system.

The receiver in this communication system should be able to decode the compound signals from multiple transmitters in order to realize the MPR capability. Many receivers based on MUD techniques exist, which are currently capable of decoding multiple signals and maximizing the signal-to-interference-plus-noise ratio (SINR) of each signal. These MUD techniques make it possible to decode the compound signals from multiple transmitters at the receiver side $[1,22]$.

For the received signal in (1), the constellation of the received signal has a number of densely distributed points for the signals received from multiple transmitters. In this case, if the constellation points of received signal from multiple transmitters are considerably contiguous or overlapped with each other, it would be quite difficult that the receiver correctly separates and identifies each signal from the compound signal. For example, Figure 2 shows the two-user constellations at 8PSK modulations when $p_{i}=1$ and $\theta_{i}=0$ for $i=1,2$. In this figure, only 33 out of 64 constellation points are visible, since the other 31 points overlap and are canceled out. Accordingly, the receiver cannot correctly separate each signal from the compound signals due to the overlapped constellation points, which are then identified as decoding errors.

According to the above communication environment, many constellations sent by multiple signal sets from multiple transmitters are distributed to the same signal space; consequently, the minimum Euclidean distance between the constellations is decreased or many constellations are canceled by overlapping. In this manner, the overlapped constellations are incorrectly demapped in the demapper; 
as a result, the transmitted signal is identified as an error. In other words, the network capacity in the multiuser communication system is not maximized, since the error probability of multiple signals is increased according to the short minimum Euclidean distance. To overcome this problem, the Euclidean distances between constellation points at the receiver side should be kept as large as possible by adjusting the carrier phase offsets at the transmitter side. Specifically, the minimum Euclidean distance between the constellation points should be maximized in order to decrease the error probability in multiple signal decoding for MPR communication systems.

In addition, the RF signals of all transmitters propagate through a wireless medium at different channel gains. Thus, even though the transmitters transmit at the same transmit power level, the power levels of the received signals would be different due to variations in the channel gains. As an example, suppose that two transmitters exist (i.e., $s_{1}$ and $s_{2}$ ) that have the highest and the weakest received signal power level $\left(p_{1}\right.$ and $\left.p_{2}\right)$, respectively. When the received signal power level of $s_{2}\left(p_{2}=0.3\right)$ is much smaller than that of $s_{1}\left(p_{1}=1\right)$, the minimum Euclidean distance is determined by that between $s_{2}$ 's own constellation points, as shown in Figure 3a. In contrast, when $p_{2}$ is set to 0.7, as depicted in Figure $3 \mathrm{~b}$, the minimum Euclidean distance is determined by $p_{1}$ of $s_{1}$ as well as $p_{2}$ of $s_{2}$. This condition implies that the transmit power levels for simultaneously transmitting users should be properly coordinated to ensure performance improvements in MPR communication systems.

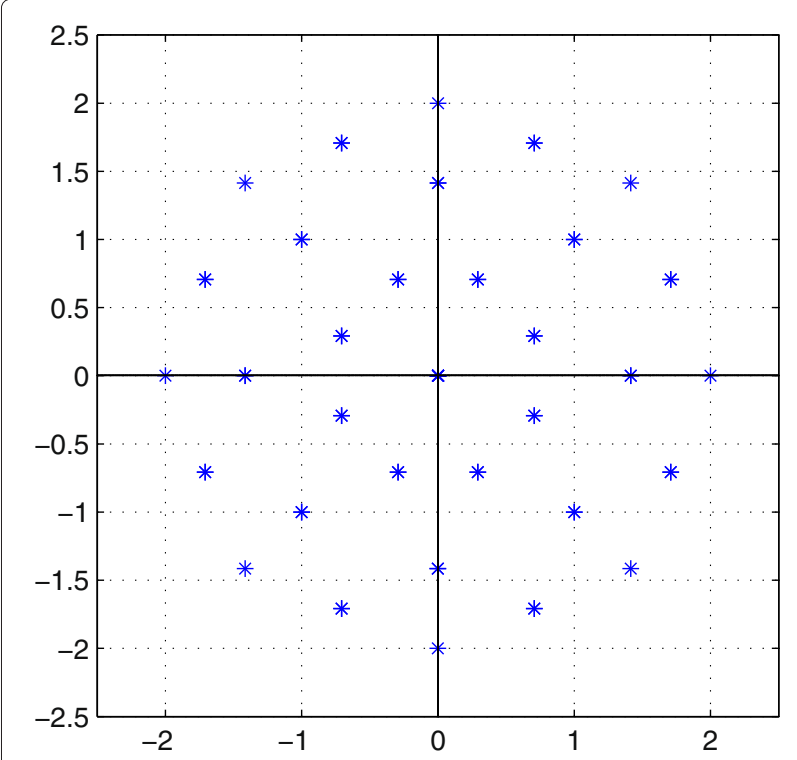

Figure 2 Motivation of carrier phase adjustment. Two-user constellations for 8PSK modulation signal sets (31 constellation points are overlapped with other points).

\section{Proposed transmit power and carrier phase adjustment scheme}

In this section, we propose a feedback-based transmit power and carrier phase adjustment scheme that controls the transmit power level and carrier phase offset in order to fully exploit the MPR channel capacity. The proposed transmit power and carrier phase adjustment scheme has two steps. The first is the carrier phase estimation, which estimates the carrier phase offset incurred by channel noises, such as AWGN and phase noise. The second is the optimal transmit power and carrier phase adjustment, which computes the optimal transmit power level and carrier phase offset, and feeds the informationwhich includes the optimal transmit power levels and carrier phase shifts-back to the transmitters as described in Figure 1. The optimal transmit power levels and carrier phase offsets are obtained for a given modulation scheme based on the placement of constellation points that maximize the minimum Euclidean distance between the points.

\subsection{Feedback scheme}

As briefly addressed in Section 2 and depicted in Figure 1, the transmitters waiting to send packets are required to transmit an RTS frame to the receiver. When the receiver detects and decodes the RTS frame signals received from the multiple transmitters, it estimates the received signal power levels and the carrier phase offsets that occur when the compound signals are transmitted on a wireless medium. Under the assumption that the training sequence of RTS frames is a form of pseudo-noise (PN) sequence which is orthogonal to each other, the receiver can estimate the received signal power levels and the carrier phase offsets without signal interference among multiple transmitters. Then, the receiver computes the optimal received power level and carrier phase shift so as to reduce the error probability, and returns the feedback information through a CTS frame which includes the optimal transmit power levels and carrier phase shifts. In this case, the optimal transmit power can be calculated from the optimal received power by using the known channel gain. The carrier phase shift is then the difference between the estimated carrier phase offset of the received signal and the optimal carrier phase offset. This feedback-based transmit power and carrier phase adjustment mechanism makes it possible for the receiver to separate the constellations of multiple signals, significantly reducing the error probability at the receiver side.

In other words, the receiver estimates the received signal power level and carrier phase distortion through the RTS frame, and then broadcasts a CTS frame that includes the set of transmitters that are permitted to transmit and the transmit power level and phase shift information, 
(a)

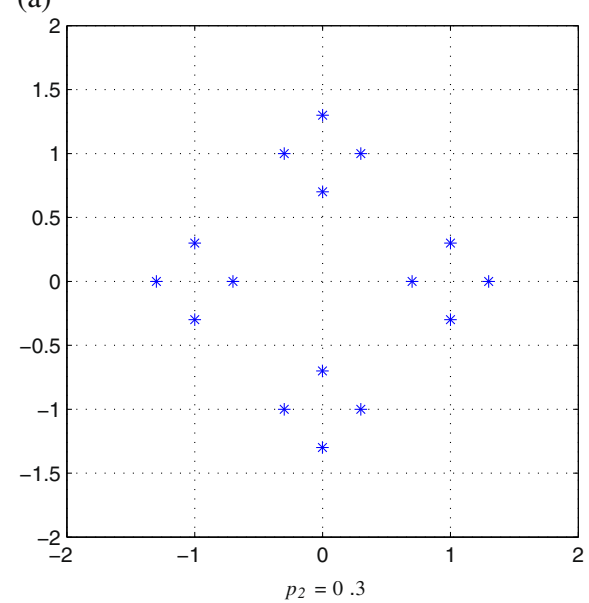

(b)

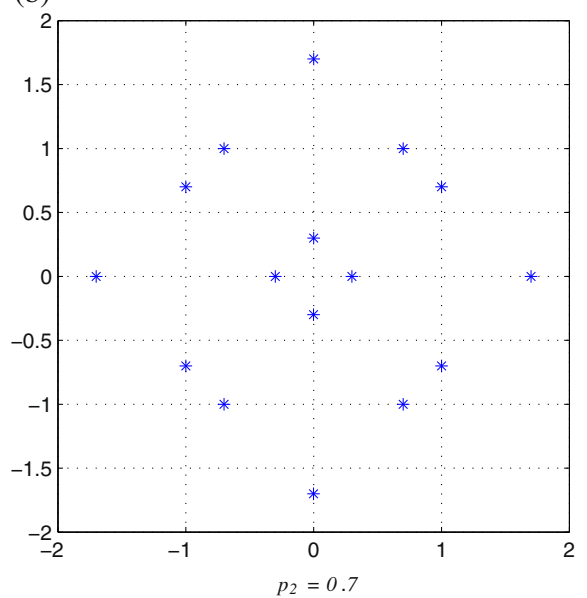

Figure 3 Motivation of power control. Two-user QPSK signal constellations with (a) $p_{2}=0.3$ and (b) $p_{2}=0.7\left(p_{1}=1\right.$ and $\theta_{i}=0$, for $\left.i=1,2\right)$.

thereby achieving low transmission error probability and high reliability.

\subsection{Transmit power level and carrier phase offset for multiple transmitters}

We propose an optimization-based approach for deriving the optimal transmit power levels and carrier phase offsets for multiple transmitters. As noted in Section 2, the minimum Euclidean distance between multiple constellations have a critical effect on the decoding performance of a multiuser wireless communication system. To minimize the bit error rate on the iterative decoding process, the minimum Euclidean distance between the received multiple constellation points should be maximized so that the receiver can successfully separate each transmitter's signal from the original superimposed signal. In this section, we derive the optimal transmit power levels and carrier phase offsets between multiple transmitters in order that the resulting transmit power and carrier phase information can be used for the optimal transmit power and carrier phase adjustment on each transmitter side.

\subsubsection{Two-user Case for QPSK Signal Set}

In order to determine the optimal transmit power levels and carrier phase offsets, we first consider an analytic derivation of the two-user QPSK signal set in a closed form. Figure 4 shows the two-user constellation for the QPSK modulation signal set. Here, we assume that the received power $\left(p_{1}\right)$ and the carrier phase of the first transmitter $\left(\theta_{1}\right)$ are 1 and 0 , respectively. Let $p_{2}$ and $\theta_{2}$ denote the received power and carrier phase of the second transmitter, respectively. Note that the range of $\theta_{2}$ is limited to be within $-\frac{\pi}{4}$ and $\frac{\pi}{4}$ in the QPSK modulation case.
In Figure 4, the adjacent constellation points $(A, B, C$, and $D$ ) are determined and depicted. Then, the coordinates of the constellation points are given by

$$
\begin{aligned}
A & =\left(p_{2} \cos \theta_{2}, 1+p_{2} \sin \theta_{2}\right), \\
B & =\left(p_{2} \sin \theta_{2}, 1-p_{2} \cos \theta_{2}\right), \\
C & =\left(1-p_{2} \cos \theta_{2},-p_{2} \sin \theta_{2}\right), \\
D & =\left(1-p_{2} \sin \theta_{2}, p_{2} \cos \theta_{2}\right),
\end{aligned}
$$

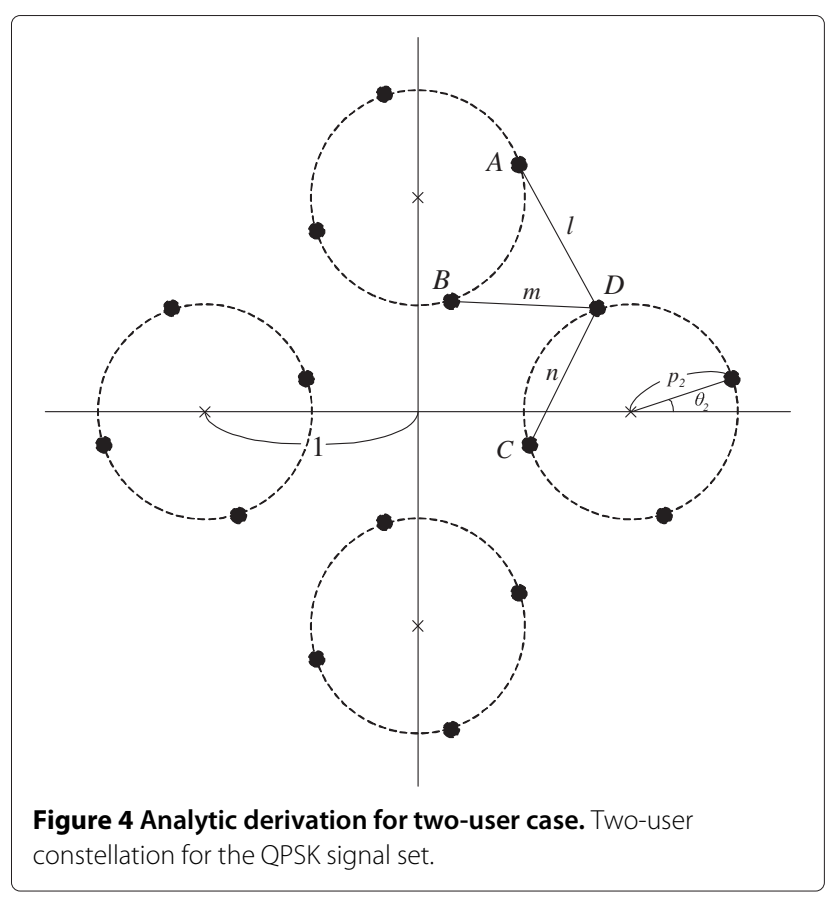


Next, let $l, m$, and $n$ denote the distances from $D$ to $A, B$, and $C$, respectively. These distances are represented by

$$
\begin{aligned}
l & =\|D-A\|, \\
m & =\|D-B\|, \\
n & =\|D-C\| .
\end{aligned}
$$

Then, the minimum Euclidean distance $\left(d_{\min }\right)$ of the two-user constellation for the QPSK modulation signal set is given as follows:

$$
d_{\min }=\min (l, m, n)
$$

For $0 \leq \theta_{2} \leq \frac{\pi}{4}$, it can be easily shown that the minimum Euclidean distance $d_{\min }$ is maximized when $l, m$, and $n$ are the same. That is, the received power $\left(p_{2}\right)$ and carrier phase $\left(\theta_{2}\right)$ of the second transmitter that maximizes the minimum Euclidean distance are obtained when $l=m=n$. By (2) and (3), the distances of $l, m$, and $n$ are as follows:

$$
\begin{aligned}
l & =\sqrt{2-4 p_{2} \cos \theta_{2}+2 p_{2}^{2}}, \\
m & =\sqrt{2-4 p_{2}\left(\sin \theta_{2}+\cos \theta_{2}\right)+4 p_{2}{ }^{2}}, \\
n & =\sqrt{2} p_{2} .
\end{aligned}
$$

To find $p_{2}$ and $\theta_{2}$, we solve the above simultaneous equations and obtain $\sin \theta_{2}=\frac{p_{2}}{2}$ and $p_{2}=\frac{1}{2 \cos \theta_{2}}$. Finally, we derive the optimal received power level and carrier phase offset as follows:

$$
p_{2}=0.5176 \text { and } \theta_{2}=\frac{\pi}{12} .
$$

In the case of more than two transmitters, it becomes more complicated to derive the optimal received power and carrier phase. Therefore, we solve an optimization problem numerically in order to determine the optimal values for multiple transmitters.

\subsubsection{Optimization of transmit power and carrier phase for multiple transmitters}

As stated in Section 2, $y$ is the received compound signals from multiple transmitters for $M$-PSK modulation and is represented in (1). If we let $C_{M, N}$ denote a set of the constellation points with $N$ transmitters for $M$ PSK modulation, the received compound signals $y$ from $N$ transmitters for $M$-PSK modulation has $M^{N}$ constellation points, as briefly explained in Section 2; i.e., $\left|\boldsymbol{C}_{M, N}\right|=$ $M^{N}$. Note that illustrative examples of $C_{4,2}$ and $C_{8,2}$ are shown in Figure 5.

The optimal received power levels and carrier phase offsets are obtained by the placement of constellation points when the minimum Euclidean distance between the points are maximized. Therefore, we formulate the following optimization problem to determine these values:

$$
\begin{aligned}
& \operatorname{maximize}_{\boldsymbol{p}, \boldsymbol{\theta}}\left(\min _{\substack{r, s \in \mathcal{C}_{M, N} \\
r \neq s}} d(r, s)\right) \\
& \begin{array}{ll}
\text { subject to } & 0<p_{i} \leq p_{i}^{\max } \\
& \theta_{i} \in\left[0, \frac{\pi}{M}\right]
\end{array} \text {, for } i=1, \cdots, N,
\end{aligned}
$$

where $d(r, s)$ is the Euclidean distance among the constellation points $r$ and $s$ in the set of $C_{M, N}$, i.e., $d(r, s)=$ $\|r-s\|, \boldsymbol{p}$ and $\boldsymbol{\theta}$ are sets of the received power levels and carrier phase offsets, respectively, $N$ is the number of transmitters that transmitted a signal, and $p_{i}^{\max }$ is the maximum received power level. Note that the above optimization maximizes the minimum Euclidean distance for all pairs of transmitters.

We then numerically solve the optimization for 2-4 transmitters for QPSK and 8PSK modulations. This optimization problem to find the optimal power and phase of multiple users is an NP-hard and nonlinear problem. Here, we use the sequential quadratic programming (SQP) method which is a useful method for numerically solving constrained nonlinear optimization problems. The SQP method iteratively solves a quadratic programming (QP) subproblem and updates an estimate by using the solution of QP subproblem at each iteration. The results represent the optimal received power level and carrier phase offset of each transmitter (rounded to five decimal places), and are listed in Table 1. In this case, the optimal values of all



Figure 5 Optimized result of two-user 8PSK. Two-user constellations for 8PSK modulation signal sets $\left(\boldsymbol{C}_{8,2}\right)$ under the proposed transmit power and carrier phase adjustment scheme. 
Table 1 Optimal received power levels and carrier phase offsets

\begin{tabular}{lcccc}
\hline $\begin{array}{l}\text { Number of } \\
\text { transmitters }\end{array}$ & Modulation & $\boldsymbol{p}_{\mathbf{2}}^{*}$ & $\boldsymbol{p}_{\mathbf{3}}^{\boldsymbol{*}}$ & $\boldsymbol{p}_{\mathbf{4}}^{\boldsymbol{*}}$ \\
\cline { 3 - 5 } & & $\boldsymbol{\theta}_{\mathbf{2}}^{\boldsymbol{*}}$ & $\boldsymbol{\theta}_{\mathbf{3}}^{\boldsymbol{*}}$ & $\boldsymbol{\theta}_{\mathbf{4}}^{*}$ \\
\hline 2 & QPSK & 0.5176 & - & - \\
& & 0.2618 & - & - \\
& 8PSK & 0.5668 & - & - \\
& & 0.0319 & - & - \\
& & 1.0000 & 0.9487 & - \\
& QPSK & 0.6435 & 0.3218 & - \\
& & 0.9921 & 0.7560 & - \\
& 8PSK & 0.3261 & 0.0000 & - \\
& & 0.7181 & 0.5000 & 0.3445 \\
& QPSK & 0.5419 & 0.2403 & 0.2428 \\
& & 0.8454 & 0.6353 & 0.5000 \\
& 8PSK & 0.1821 & 0.1220 & 0.1715 \\
\hline
\end{tabular}

transmitters are normalized by that of the first transmitter, with the received power level and carrier phase offset of the first transmitter being 1 and 0 , respectively. Note that the optimal received power level and carrier phase offset for the two-user QPSK case obtained by optimization are equal to that by derivation in (5). As an illustrative example, the two-user constellations for the 8PSK modulations are then shown in Figure 5. Using the obtained results, all 64 constellation points for the 8PSK modulations are non-overlapped with the minimum Euclidean distance maximized, unlike the constellations shown in Figure 2.
3.3 Numerical evaluation of minimum Euclidean distance We then analyzed the effects of the received power level and carrier phase offset on the minimum Euclidean distance. Figure 6 shows $d_{\min }$ for the two-user QPSK and 8PSK signal cases with respect to the received power level and carrier phase offset of the second transmitter. In this case, the signal power level and carrier phase offset of the first transmitter are respectively, fixed at 1 and 0 . The range of second transmitter's signal power level $\left(p_{2}\right)$ varies from 0 to 1 , and the carrier phase offset $\left(\theta_{2}\right)$ range varies from 0 to $90^{\circ}$.

Figure 6a shows the value of $d_{\min }$ for the two-user QPSK signal case, which is symmetric with respect to the point at which the second transmitter's carrier phase offset is $\frac{\pi}{4}$. Note that the signal set is applied to the QPSK modulation. The maximum value of $d_{\min }$ is obtained when $p_{2}=0.5176$ and $\theta_{2}=15^{\circ}(0.2618)$. As the signal power of the second transmitter varies from 0 to $0.5, d_{\min }$ increases with respect to $p_{2}$ because the minimum value of $d_{\min }$ is determined by the Euclidean distance between the constellation points corresponding to the same group of the second transmitter. After the value of $0.5, d_{\min }$ decreases because the constellation points corresponding to different groups are getting closer to each other. Figure $6 \mathrm{~b}$ shows $d_{\min }$ for the two-user 8PSK signal case. Similar to the QPSK case, the value of $d_{\min }$ is symmetric with respect to the point at which the second user's carrier phase offset is $\frac{8}{\pi}$. The maximum $d_{\min }$ in this case is obtained when $p_{2}=0.5668$ and $\theta_{2} \approx 1.83^{\circ}(0.0319)$.

We compare the performance of the proposed scheme in two-user QPSK case with that of the single-user 16PSK case. Since the number of joint constellation points for two-user QPSK case is the same as the number of


Figure 6 Numerical evaluation of $\boldsymbol{d}_{\mathbf{m i n}}$. Minimum Euclidean distance $\left(d_{\mathrm{min}}\right)$ with respect to the received power level and carrier phase offset of the second user for (a) QPSK and (b) 8PSK modulation. 
constellation points for single-user 16PSK case, the two cases have the same sum rate. Therefore, we analyze the performance of two cases in terms of the minimum Euclidean distance. In general, $d_{\mathrm{min}}$ of single-user M-PSK is given by

$$
d_{\min }=\sqrt{2 E_{s}\left(1-\cos \frac{2 \pi}{M}\right)},
$$

where $E_{s}$ is the symbol energy. To simplify the analysis, we assume that the symbol energy is 1 . Under this assumption, $d_{\text {min }}$ of single-user 16PSK is $\sqrt{2(1-\cos (2 \pi / 16)} \approx$ 0.3902 . The probability of bit error for single-user 16PSK $\left(P_{e, 16 \mathrm{PSK}}\right)$ is as follows:

$$
\begin{aligned}
P_{e, 16 \mathrm{PSK}} & =Q\left(\sqrt{\frac{d_{\mathrm{min}}^{2}}{2 N_{0}}}\right)=Q\left(\frac{0.3902}{\sqrt{2 N_{0}}}\right) \\
& =\frac{1}{2} \operatorname{erfc}\left(\frac{0.3902}{2 \sqrt{N_{0}}}\right) .
\end{aligned}
$$

In the proposed scheme, we obtain the optimal power level and phase offset $\left(p_{1}=1, p_{2}=0.5176, \theta_{1}=0^{\circ}\right.$, and $\left.\theta_{2}=15^{\circ}\right)$ for the two-user QPSK. For comparison under the same conditions as the previous single-user 16PSK, we adjust the sum of two users' power level to 1 . The power levels of the first user and second user are 0.6589 and 0.3411 , respectively. In this case, $d_{\min }$ of joint constellation points for two-user QPSK signal set is about 0.4823 . Then, the probability of bit error for two-user QPSK ( $\left.P_{e, 2-\mathrm{QPSK}}\right)$ is as follows:

$$
P_{e, 2-\mathrm{QPSK}}=\frac{1}{2} \operatorname{erfc}\left(\frac{0.4823}{2 \sqrt{N_{0}}}\right) .
$$

If the noise condition is the same, $P_{e, 16 \mathrm{PSK}}$ is higher than $P_{e, 2-\mathrm{QPSK}}$. That is, the proposed scheme for two-user QPSK case is more efficient than the single-user 16PSK case, even though two cases have the same number of constellation points and the same sum rate.

\section{Performance evaluation}

We conducted a performance evaluation for the proposed transmit power and carrier phase adjustment scheme through a comparison with an unmodified PSK modulation scheme. We implemented the optimal transmit power and carrier phase adjustment system for MPR depicted in Figure 1, which included the successive interference cancelation, demapper, decoder, signal power and carrier phase estimator, and the feedback scheme for the optimal transmit power levels and carrier phase offsets. Two modulation schemes (QPSK and 8PSK) were evaluated, and the number of transmitters was varied from 2 to 4 . Note that the carrier phase was uniformly distributed from 0 to $2 \pi$, and the bit error rate (BER) performance was evaluated with respect to the SNR on the AWGN channel.

Figure 7a shows the BERs of the proposed transmit power and carrier phase adjustment scheme in comparison with the no adjustment case for QPSK modulation with respect to the SNR and number of transmitters (i.e., the number of distinct signals that are compounded into the received signal at the receiver side). In this figure, the proposed scheme gives the lower BER values than the unmodified QPSK over the entire SNR range. The proposed scheme shows a gain of about $5 \mathrm{~dB}$ at a BER of $10^{-4}$. Figure $7 \mathrm{~b}$ then shows the BER performance of the proposed scheme and the unmodified 8PSK modulation.

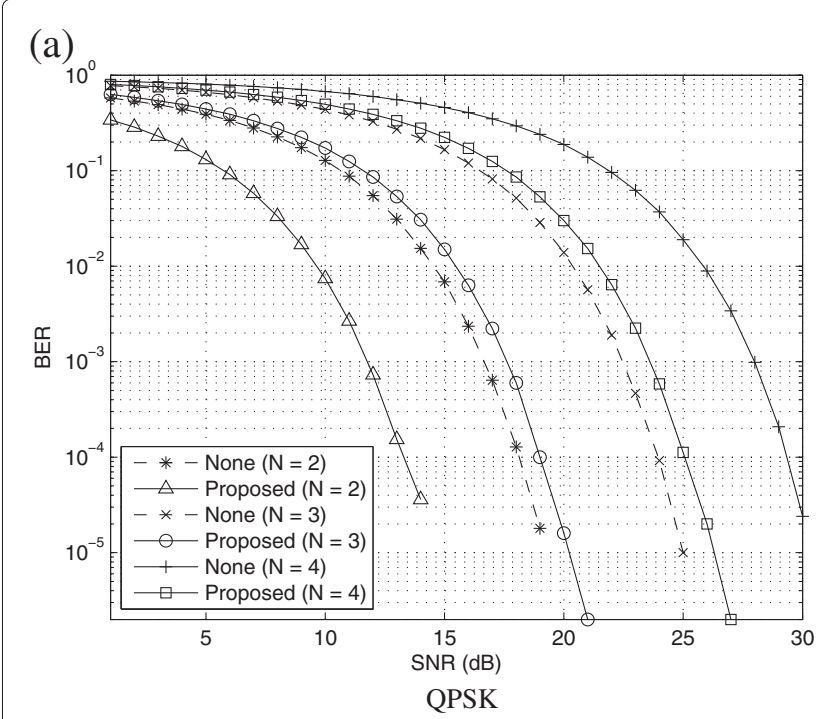

(b)



Figure 7 BER performance. BER performance for (a) QPSK and (b) 8PSK modulation. 


\section{(a)}

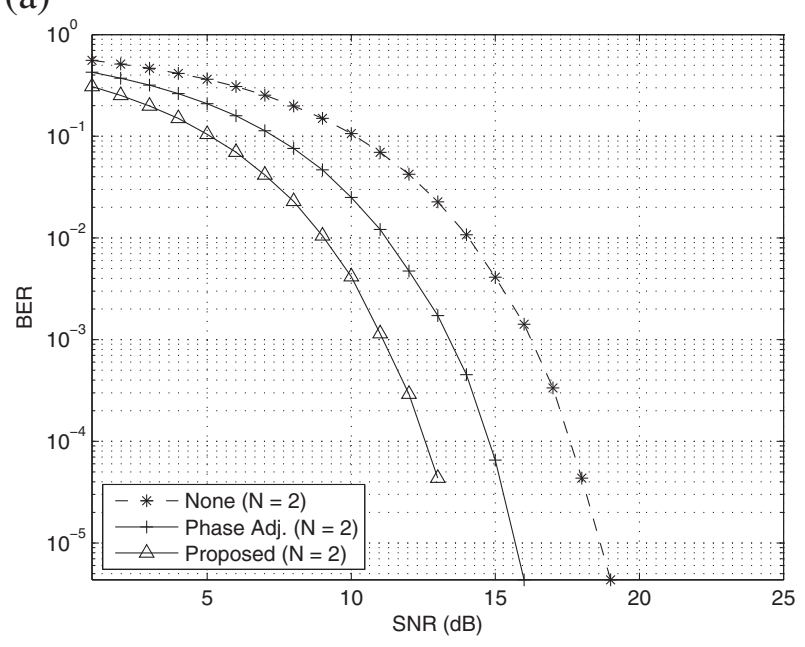

QPSK (b)

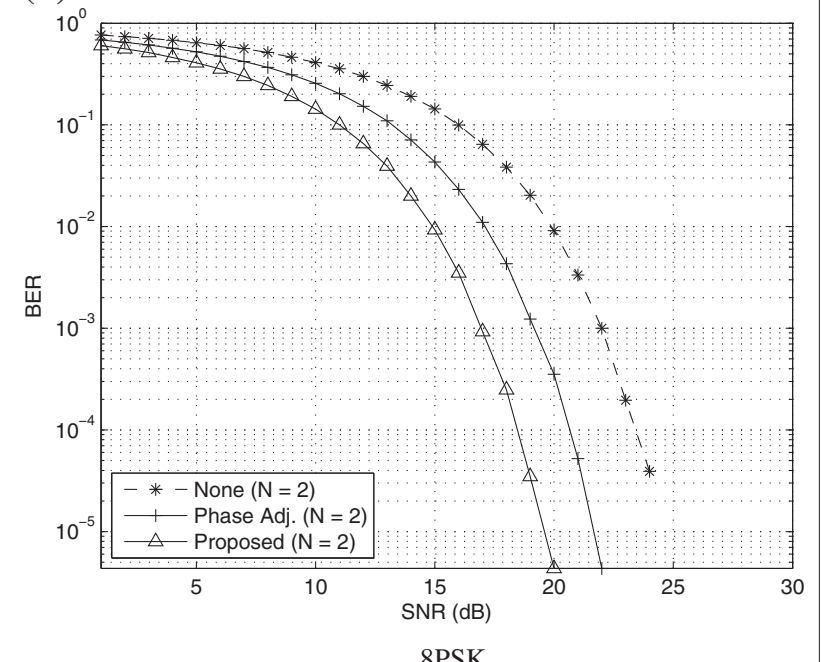

8PSK

Figure 8 BER performance comparison. BER performance comparison of the proposed scheme with the previous study (Phase Adj.) [10] for (a) QPSK and (b) 8PSK modulation.

As like the previous results for the QPSK modulation, for all cases, the BER performance of proposed scheme has much lower values than the unmodified 8PSK. We obtained an SNR gain of almost $5 \mathrm{~dB}$ with two transmitters at a BER of $10^{-4}$.

Figure 8 presents the comparison of BER performance between the proposed scheme and the carrier phase adjustment scheme in [10]. Note that the scheme in [10] adjusts only the carrier phase offset of the transmitters without modifying their transmit powers under the assumption that the received power levels from multiple transmitters are the same. As shown in Figure $8 \mathrm{a}, \mathrm{b}$ the proposed scheme gives the lower BER values than their previous study in the entire range of SNR. These comparison results imply that the coordination of both transmit powers and carrier phases among multiple transmitters can achieve the better performance than in the case that only the carrier phases are adjusted.

These results imply that the proposed scheme effectively adjusts the transmit power levels and carrier phase offsets of transmitters so that the signals from multiple transmitters are well separated over a wide range of carrier phase error variations, and that the MPR capability is fully utilized.

\section{Conclusion}

In this article, we proposed a feedback-based optimal transmit power and carrier phase adjustment scheme in order to fully take advantage of the MUD technique in MPR-capable wireless networks by coordinating the transmit power and carrier phase of each transmitter.
To determine the optimal constellation placement of the compound signals at the receiver, we formulated an optimization problem and then numerically obtained the optimal transmit power levels and carrier phase offsets for 2-4 transmitters for M-PSK modulation. Under the proposed scheme, the compound signals from multiple transmitters became more separable on its constellation at the receiver; as a result, the BER performance significantly improved in comparison with the no adjustment cases.

As future study, we plan to implement MPR-capable wireless communications based on this transmit power and carrier phase adjustment scheme on softwaredefined-radio (SDR) to obtain an empirical evaluation.

\section{Endnote}

${ }^{\text {a }}$ The information containing the optimal transmit power levels and carrier phase shifts requires only a small number of bits, thus the overhead for the feedback information in the CTS frame is negligible.

\section{Competing interests}

The authors declare that they have no competing interests.

\section{Acknowledgements}

This study was supported by Leading Foreign Research Institute Recruitment Program through the NRF funded by the MEST

(K20901002277-12E0100-06010), by the WCU program by the MEST of Korea (R31-10026), and by the GIST basic research project.

\section{Author details}

${ }^{1}$ Gwangju Institute of Science and Technology, Gwangju 500-712, Republic of Korea. ${ }^{2}$ Telecommunications Technology Association (TTA), Seongnam, Gyonggi-do 463-824, Republic of Korea. ${ }^{3}$ School of Information and Communication Engineering, Inha University, Incheon 402-751, Republic of 
Korea. ${ }^{4}$ Department of Nanobio Materials and Electronics, Gwangju Institute of Science and Technology, Gwangju 500-712, Republic of Korea.

Received: 31 January 2012 Accepted: 10 December 2012

Published: 2 January 2013

\section{References}

1. S Verdú, Multiuser Detection. (Cambridge University Press, Cambridge, UK, 1998)

2. S Verdú, Minimum probability of error for asynchronous Gaussian multiple-access channels. IEEE Trans. Inf. Theory. 32(1), 85-96 (1986)

3. R Lupas, S Verdú, Linear multiuser detectors for synchronous code-division multiple-access channels. IEEE Trans. Inf. Theory. 35(1), 123-136 (1989)

4. A Duel-Hallen, Decorrelating decision-feedback multiuser detector for synchronous code-division multiple-access channel. IEEE Trans. Commun. 41(2), 285-290 (1993)

5. U Madhow, M Honig, MMSE interference suppression for direct-sequence spread-spectrum CDMA. IEEE Trans. Commun. 42(12), 3178-3188 (1994)

6. MK Varanasi, B Aazhang, Multistage detection in asynchronous code-division multiple-access communications. IEEE Trans. Commun. 38(4), 509-519 (1990)

7. M Honig, U Madhow, S Verdú, Blind adaptive multiuser detection. IEEE Trans. Inf. Theory. 41(4), 944-960 (1995)

8. S Ulukus, RD Yates, A blind adaptive decorrelating detector for CDMA systems. IEEE J. Sel. Areas Commun. 16(8), 1530-1541 (1998)

9. XWang, HV Poor, Blind adaptive multiuser detection in multipath CDMA channels basedon subspace tracking. IEEE Trans. Sig. Process. 46(11), 3030-3044 (1998)

10. W Choi, T Kim, H Lee, H Lim. Carrier phase adjustment for multiple acces communication systems with multi-packet reception capability, in Proc. IEEE WCNC, (Sydney, Australia, 2010), pp. 1-6

11. S Ghez, S Verdú, S Schwartz, Stability properties of slotted Aloha with multipacket reception capability. IEEE Trans. Autom. Control. 33(7), 640-649 (1988)

12. D Tse, S Hanly, Linear multiuser receivers: effective interference, effective bandwidth and user capacity. IEEE Trans. Inf. Theory. 45(2), 641-657 (1999)

13. L Collin, O Berder, P Rostaing, G Burel, Optimal minimum distance-based precoder for MIMO spatial multiplexing systems. IEEE Trans. Signal Process. 52(3), 617-627 (2004)

14. L Tong, Q Zhao, G Mergen, Multipacket reception in random access wireless networks: From signal processing to optimal medium access control. IEEE Commun. Mag. 39(11), 108-112 (2001)

15. W Choi, D Jung, H Lee, H Lim. Power control for multiple access communication systems with multi-packet reception capability, in Proc. IEEE LCN, (Zurich, Switzerland, 2009), pp. 281-284

16. WF Yang, JY Wu, LC Wang, TS Lee, A multigroup priority queueing MAC protocol for wireless networks with multipacket reception. EURASIP J. Wirel. Commun. Network. 16, 1-12 (2008)

17. H Steendam, N Noels, M Moeneclaey. Iterative carrier phase synchronization for low-density parity-check coded systems, in Proc. IEEE ICC, (Anchorage, USA, 2003), pp. 3120-3124

18. L Zhang, A Burr, Iterative carrier phase recovery suited to turbo-coded systems. IEEE Trans. Wirel. Commun. 3(6), 2267-2276 (2004)

19. J Harshan, B Rajan. Finite signal-set capacity of two-user Gaussian multiple access channel, in Proc. IEEE ISIT, (Toronto, Canada, 2008), pp. 1203-1207

20. J Harshan, B Rajan. Coding for two-user Gaussian MAC with PSK and PAM signal sets, in Proc. IEEE ISIT, (Seoul, Korea, 2009), pp. 1859-1863

21. R Mudumbai, J Hespanha, U Madhow, G Barriac, Distributed transmit beamforming using feedback control. IEEE Trans. Inf. Theory. 56(1), 411-426 (2009)

22. S Ulukus, RD Yates, Adaptive power control and MMSE interference suppression. Springer-Wirel. Netw. 4(6), 489-496 (1998)

doi:10.1186/1687-1499-2013-1

Cite this article as: Choi et al:: Coordinating transmit power and carrier phase for wireless networks with multi-packet reception capability. EURASIP Journal on Wireless Communications and Networking 2013 2013:1.

\section{Submit your manuscript to a SpringerOpen ${ }^{\circ}$ journal and benefit from:}

- Convenient online submission

- Rigorous peer review

- Immediate publication on acceptance

- Open access: articles freely available online

- High visibility within the field

- Retaining the copyright to your article

Submit your next manuscript at $\boldsymbol{\wedge}$ springeropen.com 